Original article

\title{
Perfluorinated compounds in blood of textile workers and barbers
}

\author{
Cheng Lu ${ }^{\mathrm{a}, \mathrm{b}}$, Ya-Li Shi ${ }^{\mathrm{b}}$, Zhen Zhou ${ }^{\mathrm{b}}$, Nan-Nan Liu ${ }^{\mathrm{b}}$, Zhao-Fu Meng ${ }^{\mathrm{a}, *}$, Ya-Qi Cai ${ }^{\mathrm{b}}$ \\ ${ }^{a}$ Department of Environmental Engineering, College of Resource and Environment, Northwest A E F University, Yangling 712100, China \\ ${ }^{\mathrm{b}}$ State Key Laboratory of Environmental Chemistry and Ecotoxicology, Research Center for Eco-Environmental Sciences, Chinese Academy of Sciences, Beijing \\ 100085, China
}

\section{A R T I C L E I N F O}

\section{Article history:}

Received 31 December 2013

Received in revised form 18 January 2014

Accepted 26 February 2014

Available online 15 March 2014

\section{Keywords:}

Perfluorinated compounds

Blood

Textile workers

Barbers

\begin{abstract}
A B S T R A C T
12 perfluorinated compounds ( $\mathrm{PFCs}$ ) in human blood from workers in a textile mill in Shandong province and several barbershops in Tianjin were analyzed in this study. It was found that perfluorooctanesulfonate (PFOS) and perfluorooctanoate (PFOA) were the most prominent PFCs, with average concentrations of $5.73 \mu \mathrm{g} / \mathrm{L}$ and $5.46 \mu \mathrm{g} / \mathrm{L}$ for textile workers, and $2.55 \mu \mathrm{g} / \mathrm{L}$ and $2.84 \mu \mathrm{g} / \mathrm{L}$ for barbers. PFOS and perfluorohexanesulfonate (PFHxS) concentrations revealed a positive correlation in blood samples $(p<0.01)$, and concentrations among PFOS, perfluorononanoic acid (PFNA) and perfluorodecanoic acid (PFDA) also revealed positive correlations $(p<0.01)$. The influence of gender and age on PFC concentration in blood was also investigated, and the results showed that there was no statistically significant difference between the male and female samples, as well as in samples from people with different ages. Generally speaking, the textile workers may face a higher exposure of PFCs than barbers.

() 2014 Zhao-Fu Meng. Published by Elsevier B.V. on behalf of Chinese Chemical Society. All rights
\end{abstract} reserved.

\section{Introduction}

Perfluorinated compounds (PFCs) are commonly used in the production of fabrics, carpets, surfactants, synthetic detergent, shampoo, perm agent series, and hairspray because of their chemical stability and water-oil resistance $[1,2]$. PFCs are chemically and physically stable due to the strong carbon-fluorine (C-F) covalent bonds, so they are difficult to degrade. What is more, some kinds of PFCs or their precursor compounds are semi-volatile, and they can travel long distances from contamination sites, making them widespread throughout the world [3,4]. Over the past decade, numerous investigations have been conducted on the exposure of PFCs in humans around the world, and concerns about the potential health consequences of PFCs have been raised due to their ubiquitous distribution. After decades of development, many researchers all over the world have reported that perfluorooctanesulfonate (PFOS) and perfluorooctanoate (PFOA) can be found in blood of local people [5-7]. In recent years, people have paid more attention to the occupational exposure of PFCs. For example, Holzer et al. [8] have reported that PFOS concentration in blood plasma of

\footnotetext{
* Corresponding author.

E-mail address: caiyaqi@rcees.ac.cn (Z.-F. Meng).
}

anglers who consume fish 2-3 times per month is 7 times higher than those without consumption of fish. Freberg et al. [9] found that the concentration of PFOA in the serum of 13 professional ski waxers was around 25 times higher than background level. The high serum concentration of perfluorohexanesulfonate in members of a Canadian family has been proved to be associated with the application of home carpeting [10]. Zhang et al. [11] found that the concentration of PFCs in the blood of workers in a leather factory in Wenzhou was much higher than general residents there. The highest concentration of PFOA in the blood of residents living near a fluorochemical plant of Liaoning was up to $93 \mathrm{ng} / \mathrm{mL}$ [12]. These studies show that occupationally exposed populations may face the risk of higher exposure levels of PFCs than the common population, and we should pay more attention to this issue. Studies on the occurrence and the potential health consequences of PFCs in occupationally exposed workers are highly necessary.

PFCs are widely used in the textile industry and barber businessrelated products $[1,2]$, such as fabrics, surfactants, synthetic detergent, shampoo, and hairspray, so the workers there may face more serious PFC exposure. However, the studies on the potential contamination, occupational exposure, and health risk of PFCs in both work places are limited worldwide. The aim of this study is to measure the concentration of PFCs in blood samples of textile workers and barbers for exploring the pollution characteristics of 
Table 1

Summary of the demographic information of blood samples.

\begin{tabular}{llllll}
\hline Sampling area & $\begin{array}{l}\text { Number of } \\
\text { samples }\end{array}$ & Age & $\begin{array}{l}\text { Career } \\
\text { experience }\end{array}$ & $\begin{array}{l}\text { Smoke } \\
\text { habit }\end{array}$ \\
\cline { 2 - 4 } & Male & Female & & & \\
\hline Shandong & 16 & 4 & $23-55$ & - & - \\
Tianjin & 36 & 13 & $16-50$ & $0.5-20$ years & $\begin{array}{l}0-10 \\
\text { (Cigarette)/day }\end{array}$ \\
\hline
\end{tabular}

PFCs in the blood of occupationally exposed workers. To the best of our knowledge, it is the first one to investigate PFCs in the blood of textile workers and barbers in China.

\section{Experimental}

As shown in Table 1, a textile mill in Shandong province and several barbershops in Tianjin were selected for sampling. Sixtynine blood samples were collected from volunteer donors in 2012. All of the donors agreed to participate in the investigation and to provide basic data such as age, gender, and occupational and residential history in a questionnaire. All sampling events were approved by the local municipal Center for Disease Control and Prevention (CDC). Approximately $5 \mathrm{~mL}$ of blood was collected from each donor and placed in a BD Vacutainer ${ }^{\circledR}$ tube (Becton Dickinson) with heparin sodium. All samples were stored at $-20^{\circ} \mathrm{C}$ until analysis.

The blood samples were extracted using the method developed by Yeung et al. [13]. In brief, blood samples were thawed at room temperature before extraction. $0.5 \mathrm{~mL}$ of sample was added into a $15 \mathrm{~mL}$ PP tube containing $40 \mu \mathrm{L}$ of internal standard $(50 \mu \mathrm{g} / \mathrm{L}$, ${ }^{13} \mathrm{C}_{4}$-PFOS, ${ }^{13} \mathrm{C}_{4}$-PFOA, ${ }^{13} \mathrm{C}_{4}$-PFBA and ${ }^{13} \mathrm{C}_{4}$-PFDoDA). Then, $5 \mathrm{~mL}$ of acetonitrile were added to it. After mixing thoroughly, the mixture was shaken for $20 \mathrm{~min}$ at $250 \mathrm{rpm}$ and centrifuged at $3000 \mathrm{rpm}$ for $10 \mathrm{~min}$. Then, approximately $5 \mathrm{~mL}$ of supernatant was taken out and transferred to another $15 \mathrm{~mL} \mathrm{PP}$ tube. The procedure was repeated twice as mentioned above. All three extracts were combined, and the final extract was condensed to $0.5 \mathrm{~mL}$ in the presence of nitrogen gas. Finally, the condensed extract was diluted with $10 \mathrm{~mL}$ of deionized water prior to the clean up by solid phase extraction. The dilution was loaded onto the SPE column (Oasis WAX, $150 \mathrm{mg}, 6 \mathrm{cc}$ ) preconditioned with $4 \mathrm{~mL}$ of methanolammonia (0.1\% in methanol), $4 \mathrm{~mL}$ of methanol, and $10 \mathrm{~mL}$ of Milli$\mathrm{Q}$ water. The SPE column was then washed with $4 \mathrm{~mL}$ of acetic acid $(\mathrm{pH} 4)$ and $8 \mathrm{~mL}$ of water, and then the target analyte was eluted from the column with $4 \mathrm{~mL}$ of methanol and $4 \mathrm{~mL}$ of methanolammonia $(0.1 \%$ in methanol) at a flow rate of $1 \mathrm{drop} / \mathrm{s}$. The eluate was concentrated to $1 \mathrm{~mL}$ under nitrogen for injection.

An HPLC-ESI-MS/MS system composed of a P680 pump, Ultimate 3000 auto-sampler, and Chromeleon 6.70 software was used to analyze the samples. Separation was accomplished on a Dionex Acclaim $120 \mathrm{C}_{18}$ column $(150 \mathrm{~mm} \times 4.6 \mathrm{~mm}, 5 \mu \mathrm{m})$. Mass spectrometry was conducted with a tandem mass spectrometer system (MS/MS) (API 3200, Applied Biosystems/MDS SCIEX, US) with electrospray ionization (ESI) and Analyst 1.4 .2 software. A ten-point standard calibration $(0.05,0.10,0.20,0.50,1.00,2.00$, $5.00,10.00,20.00$ and $50.00 \mu \mathrm{g} / \mathrm{L}$ ) was conducted for every batch of analysis, the deviation of every point from the regression line was less than $20 \%$ from its theoretical value. The regression coefficients $\left(r^{2}\right)$ of the calibration curve for all target analytes were higher than 0.99 . Only polypropylene (PP) tubes were adopted during the process. Ammonium acetate was purchased from Alfa Aesar, and $\mathrm{CH}_{3} \mathrm{OH}$ was of optimal grade (Fisher Scientific, Pittsburgh, PA). Mill-Q water was prepared on a Milli-Q Advantage A10 system (Millipore, USA) with a specific resistance of $18.2 \mathrm{M} \Omega \mathrm{cm}$.
Procedural blanks were conducted for every 10 samples to check for possible contamination and interference. The blood samples spiked with $5 \mathrm{ng}$ of target standards were used to evaluate the recovery. The average extraction recoveries ranged from $94.2 \%$ to $117.4 \%$, and the relative standard deviation (RSD) was $0.5-14.6$. The detection limit (DL) of this method was determined at the lowest concentration resulting in a signal-to-noise ratio $(S / N)$ of 3 , and the quantification limit (QL) of this method was determined at the lowest concentration resulting in a signal-to-noise ratio $(S / N)$ of 10. All statistical analyses were performed with SPSS 18.0 software and Microsoft Excel.

\section{Results and discussion}

Several kinds of PFCs were detected in blood samples. As shown in Fig. 1, PFOA and PFOS were the predominant PFCs, and the average concentrations of PFOS and PFOA in the blood from textile workers and barbers were similar. The average concentrations of PFOS in the blood from textile workers and barbers were 5.73 and $2.55 \mu \mathrm{g} / \mathrm{L}$ respectively, and the concentration ranges of PFOS were $1.34-14.75 \mu \mathrm{g} / \mathrm{L}$ and $0.44-7.72 \mu \mathrm{g} / \mathrm{L}$. As for PFOA, the average concentrations were 5.46 and $2.84 \mu \mathrm{g} / \mathrm{L}$, and the concentration ranges were $2.35-10.92 \mu \mathrm{g} / \mathrm{L}$ and $0.71-12.18 \mu \mathrm{g} / \mathrm{L}$, respectively. The average concentration of PFOA was slightly higher than PFOS in blood samples of barbers. In contrast, PFOS usually revealed a higher concentration than PFOA in human blood in many previous studies $[6,14,15]$. For example, our previous studies on PFCs in blood from people in China have pointed out that the average concentration of PFOS $(10.60 \mu \mathrm{g} / \mathrm{L})$ is 10 times higher than that of PFOA $(1.39 \mu \mathrm{g} / \mathrm{L})$ [14]. Olsen et al. [6] have found that the average concentration of PFOS was 20-30 $\mu \mathrm{g} / \mathrm{L}$, and PFOA was $2-5 \mu \mathrm{g} / \mathrm{L}$ in blood samples of the donors to the American Red Cross. Ji et al. [15] have reported that the average concentration of PFOS and PFOA was 3.98 and $1.37 \mu \mathrm{g} / \mathrm{L}$, respectively, in blood samples from Korea's general population. The average concentration of PFOS in our study is similar with that of the general population but the average concentration of PFOA is significantly higher. Therefore, the workers in textile mills and barbershops may have higher PFOA exposure. When compared with other occupationally exposed workers, the average concentrations of PFOS and PFOA in our study exhibited a lower level. For instance, researchers have explored PFCs in the blood of workers from a fluorochemical plant, and found that the average concentration of PFOS is $21.30 \mu \mathrm{g} / \mathrm{L}$, which is four times higher than that in our study. Similarly, the average concentration of PFOA reaches up to $1078.87 \mu \mathrm{g} / \mathrm{L}$, which is 200 times higher than that in our study [16]. Beesoon et al. [10] have reported that the concentration of PFOS in blood samples from a Canadian family associated with home carpet treatment reaches up to $23.42 \mu \mathrm{g} / \mathrm{L}$, which was four-fold higher than our result, and the concentration of PFOA is $3.16 \mu \mathrm{g} / \mathrm{L}$, which is similar with our result. Therefore, we can come to a conclusion that textile mill and barbershops have a lower risk for occupational exposure of PFCs when compared with these occupational places.

It should be noted that a significantly higher concentration of PFNA is detected in textile workers $(0.31-2.36 \mu \mathrm{g} / \mathrm{L})$ and barbers $(0.13-1.15 \mu \mathrm{g} / \mathrm{L})$. The concentration of PFNA in blood samples of textile workers is higher than that in general populations from America (0.25-0.55 $\mu \mathrm{g} / \mathrm{L})$ [17] and Sweden (0.20-0.50 $\mu \mathrm{g} / \mathrm{L})$ [18], which is similar with that in general populations from Japan $(0.20-$ $4.60 \mu \mathrm{g} / \mathrm{L})$ [19] and Korea (0.42-1.83 $\mu \mathrm{g} / \mathrm{L})$ [15]. China, Japan, and Korea have higher and similar PFNA concentrations, indicating that they may have higher application amounts and have a similar exposure pathway. The data of polyfluoroalkyl chemicals in the U.S. population provided by National Health and Nutrition Examination Survey (NHANES) has revealed the decreased serum concentrations of PFOS and PFOA, and increased serum 
(a) PFCs Composition

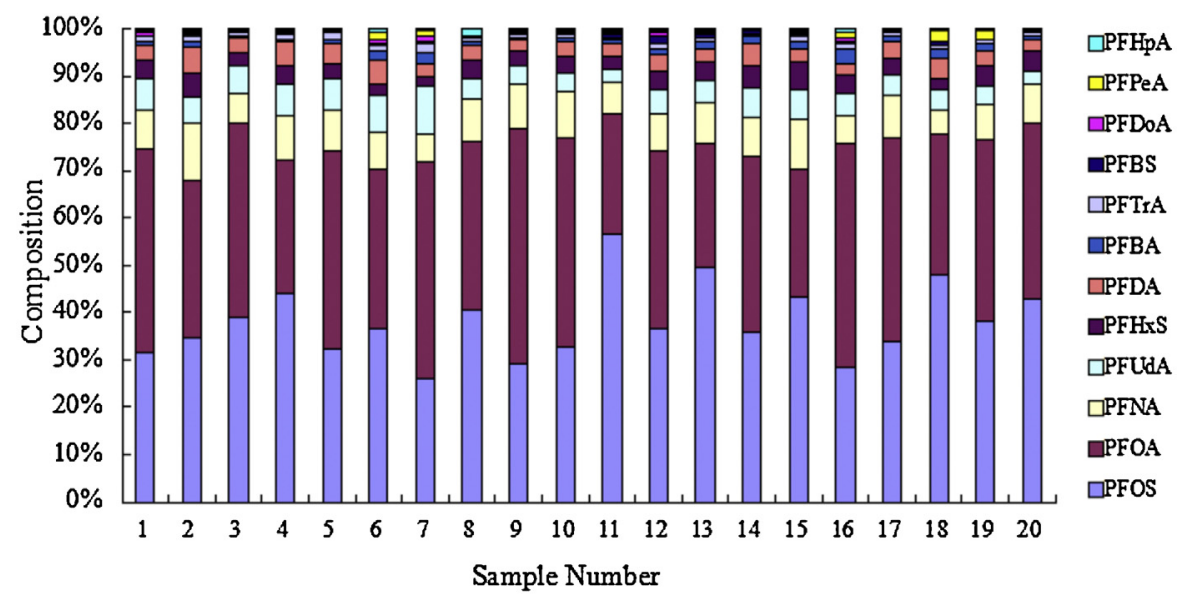

(b)

PFCs Composition

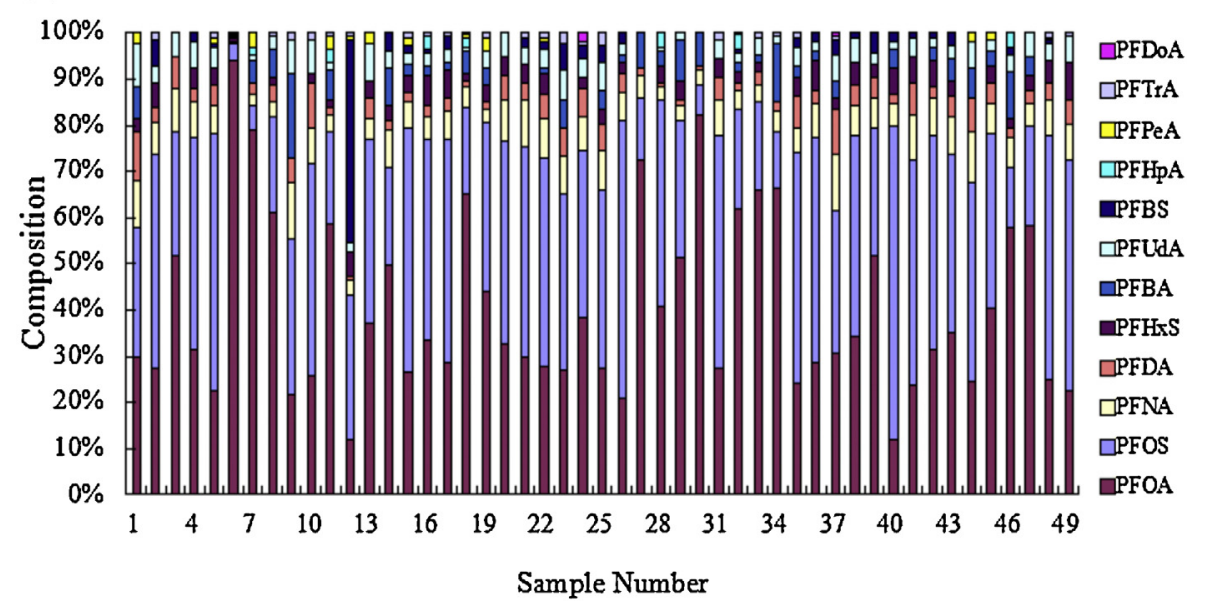

Fig. 1. The composition profiles of the PFCs detected in textile mill (a) and barbershop (b).

concentration of PFNA during the period between 1999/2000 and 2003/2004 [20]. Biodegradation of some precursors may be responsible for the increasing concentration of PFNA in the environment [20]. For example, the metabolism of 8:2 FTOH can promote the production of PFNA [15]. In addition, ammonium perfluoronanoate (APFN) manufactured worldwide may be another source of PFNA [20]. According to our study, workers in textile mills may be exposed to more PFNA-containing products, thus leading to a higher PFNA concentration in blood.

As with other PFCs, PFHxS was detected in all of the blood samples, and the average concentrations were $0.53 \mu \mathrm{g} / \mathrm{L}$ and $0.24 \mu \mathrm{g} / \mathrm{L}$ for textile workers and barbers. The average concentrations of PFDA were $0.50 \mu \mathrm{g} / \mathrm{L}$ and $0.25 \mu \mathrm{g} / \mathrm{L}$ for textile workers and barbers, respectively. Generally speaking, the average concentrations of PFOS, PFOA, PFHXS and PFDA in textile workers were two times higher than those of barbers. Therefore, the textile workers may face higher exposure of PFCs than barbers.

The composition profiles of the PFCs detected in blood are shown in Fig. 1. In general, PFOA, PFOS, and PFNA were the predominant PFCs, which accounted for more than $80 \%$ of total PFCs in human blood samples. PFOS was the largest part (39.0\%) of PFCs in blood samples from textile workers. On the other hand, for blood samples from barbers, the largest part was PFOA (47.8\%). Through correlation analysis, we have found that PFOS and PFHxS concentrations were significantly correlated with each other $(p<0.01)$, and similar correlations between them have also been reported in previous studies [17]. We suspected that these people might be exposed to similar or related sources of PFOS and PFHxS in China. Besides, PFHxS can be formed from electric fluorination of PFOS-based products [19]. The concentration of PFHxS is also positively correlated with PFOS, PFNA and PFDA $(p<0.01)$. The concentrations of PFOA and PFOS are correlated with each other in blood samples from textile workers $(p<0.01)$, but a similar relationship is not observed in blood samples from barbers, which needs to be further investigated.

Some researchers have pointed out that PFCs may not have a pattern of biological accumulation and distribution as other POPs due to their relatively weak lipophilicity. However, combined with protein in the blood and liver [21], the contents of PFCs may increase with age, which is attributed to the long half-life of PFOS and PFOA in human tissues ( $4-5$ years in serum) [22]. PFC concentrations in blood samples of our study were classified for testing the possible influence from age, but a statistically significant difference was not observed, which was in accordance with a study on PFC concentration in blood samples from 9 cities in China [23]. The possible reason is due to the fact that our sample size is limited, and age distribution is relatively narrow.

As shown in Table 2, the serum levels of PFCs with respect to gender were also investigated by using 49 samples of barbers. The 
Table 2

PFC concentrations in samples from barbershops $(\mu \mathrm{g} / \mathrm{L})$.

\begin{tabular}{|c|c|c|c|c|c|c|c|c|}
\hline \multirow[t]{2}{*}{ PFCs } & \multicolumn{4}{|c|}{ Male $(n=36)$} & \multicolumn{4}{|c|}{ Female $(n=13)$} \\
\hline & Minimum & Maximum & Mean & Median & Minimum & Maximum & Mean & Median \\
\hline PFOS & 0.44 & 7.72 & 2.56 & 2.10 & 0.88 & 6.90 & 2.19 & 2.12 \\
\hline PFOA & 0.78 & 12.18 & 3.18 & 2.60 & 0.71 & 4.80 & 1.89 & 1.39 \\
\hline PFDA & 0.06 & 0.58 & 0.22 & 0.22 & 0.09 & 0.86 & 0.29 & 0.19 \\
\hline PFHxS & 0.00 & 0.92 & 0.26 & 0.21 & 0.00 & 0.57 & 0.18 & 0.16 \\
\hline PFNA & 0.13 & 1.15 & 0.41 & 0.36 & 0.22 & 1.05 & 0.41 & 0.35 \\
\hline PFUdA & 0.00 & 0.56 & 0.21 & 0.20 & 0.10 & 0.51 & 0.24 & 0.24 \\
\hline
\end{tabular}

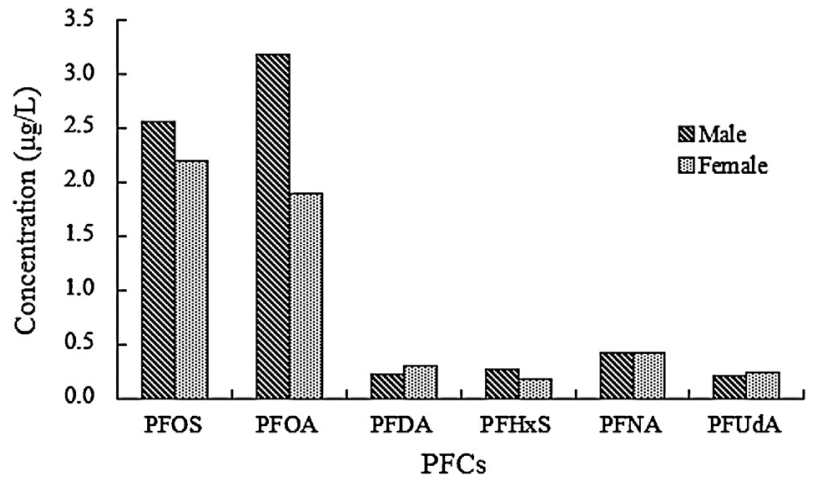

Fig. 2. Average concentration of PFCs in blood samples $(\mu \mathrm{g} / \mathrm{L})$.

PFC concentration was generally higher in males than in females (Fig. 2), while a statistically significant difference was not observed in either $(p>0.01$, two-tailed $t$-text). Previous reports have demonstrated the statistical differences in PFC levels between males and females. For example, Calafat et al. [17] have reported that the serum level of PFOS, PFOA, PFHxS, and PFNA are higher in men than in women. In blood samples of the workers in USA, males have higher average concentration of PFOS than females [11]. In contrast, in other reports, the concentration of PFCs in blood is not correlated with gender. For instance, in blood samples collected from the general population of Korea, the influence of gender on PFCs in blood was not observed [15]. Although the reason for age and gender-related difference in PFC concentrations is not clear, this may be attributed to confounding factors such as occupation, lifestyle, exposure history, and some physiological factors.

\section{Conclusion}

A large amount of knowledge in the field of environmental PFCs has been accumulated so far, while we are still just at the beginning of research into the concentration of PFCs in the blood of occupationally exposed workers in China. The present study indicates that PFCs were widespread in textile mill and barbershops. PFOS and PFOA were the major PFCs in blood samples. Gender and age influence on PFC concentrations were also studied in the present study, and no statistically significant difference between the male and female samples was observed, as well as in samples from the people with different ages. The textile workers and barbers may face a higher exposure of several kinds of PFCs than the general population, and more work is essential to define the possible exposure of PFCs and the long-term trend in the two occupational places in China.

\section{Acknowledgments}

This work was supported by the National Natural Science Foundation of China (Nos. 41023005, 21377145, 21321004) and the State Environmental Protection Welfare Scientific Research Project (No. 201009026).

\section{References}

[1] B.D. Key, R.D. Howell, C.S. Criddle, Fluorinated organics in the biosphere, Environ. Sci. Technol. 31 (1997) 2445-2454.

[2] X.L. Chen, L. Hong, H.Z. Wang, et al., Influences of PFOS directive on Chinese textile and leather industries and its countermeasures, World Stand. Qual. Manage. 10 (2009) 84-87.

[3] A.K. Sohlenius, K. Andersson, A. Bergstand, et al., Effects of perfluorooctanoic acida-a potent peroxisome proliferators in rat-on Morris hepatoma $7800 \mathrm{C} 1$ cells, a rat cell line, Biochim. Biophys. Acta 1213 (1994) 63-74.

[4] J.P. Giesy, K. Kannan, Perfluorochemical surfactants in the environment, Environ. Sci. Technol. 36 (2002) 147-152.

[5] Y.H. Jin, X. Liu, X. Zhang, et al., Perfluorooctance sufonate situation in serum of common population, Chin. J. Public Health 19 (2003) 1200-1201.

[6] G.W. Olsen, D.C. Mair, T.R. Church, et al., Decline in perfluorooctanesulfonate and other polyfluoroalkyl chemicals in America Red Cross adult blood donors, 2000 2006, Environ. Sci. Technol. 42 (2008) 4989-4995.

[7] A.M. Calafat, Z. Kuklenyik, S.P. Caudill, et al., Perfluorochemicals in pooled serum samples from United States residents in 2001 and 2002, Environ. Sci. Technol. 40 (2006) 2128-2134.

[8] J. Holzer, T. Goen, P. Just, Perfluorinated compounds in fish and blood of anglers at Lake Mohne, Sauerland area, Germany, Environ. Sci. Technol. 45 (2011) 80468052.

[9] B.I. Freberg, L.S. Haug, R. Olsen, et al., Occupational exposure to airborne perfluorinated compounds during professional ski waxing, Environ. Sci. Technol. 44 (2010) 7723-7728.

[10] S. Beesoon, S.J. Genuis, J.P. Benskin, J.W. Martin, Exceptionally high serum concentrations of perfluorohexane sulfonate in a Canadian family linked to home carpet treatment applications, Environ. Sci. Technol. 46 (2012) 12960 12967.

[11] W. Zhang, Z. Lin, M. Hu, et al., Perfluorinated chemicals in blood of residents in Wenzhou, China, Ecotoxicol. Environ. Saf. 74 (2011) 1787-1793.

[12] J. Bao, W. Liu, L. Liu, et al., Perfluorinated compounds in the environment and the blood of residents living near fluorochemical plants in Fuxin, China, Environ. Sci. Technol. 45 (2011) 8075-8080.

[13] L.W.Y. Yeung, S. Taniyasu, K. Kannan, et al., An analytical method for the determination of perfluorinated compounds in whole blood using acetonitrile and solid phase extraction methods, J. Chromatogr. A 1216 (2009) 4950-4956.

[14] Y.Y. Pan, Y.L. Shi, J.M. Wang, et al., Concentrations of perfluorinated compounds in human blood from twelve cities in China, Environ. Toxicol. Chem. 29 (2010) 2695-2701.

[15] K. Ji, S. Kim, Y. Kho, Serum concentrations of major perfluorinated compounds among the general population in Korea: dietary sources and potential impact on thyroid hormones, Environ. Int. 45 (2012) 78-85.

[16] J. Wang, Y. Zhang, W. Zhang, et al., Association of perfluorooctanoic acid with HDL cholesterol and circulating miR-26b and miR-199-3p in workers of a fluorochemical plant and nearby residents, Environ. Sci. Technol. 46 (2012) 9274-9281.

[17] A.M. Calafat, Z. Kuklenyik, J.A. Reidy, et al., Serum concentrations of 11 polyfluoroalkyl compounds in the US population: data from the National Health and Nutrition Examination Survey (NHANES) 1999-2000, Environ. Sci. Technol. 41 (2007) 2237-2242.

[18] A. Kärrman, B. van Bavel, U. Järnberg, L. Hardell, G. Lindström, Perfluorinated chemicals in relation to other persistent organic pollutants in human blood, Chemosphere 64 (2006) 1582-1591.

[19] A. Kärrman, K.H. Harada, K. Inoue, et al., Relationship between dietary exposure and serum perfluorochemical (PFC) levels - a case study, Environ. Int. 35 (2009) $712-714$.

[20] Y.X. Feng, X.M. Fang, Z.M. Shi, M.Q. Xu, J.Y. Dai, Effects of PFNA exposure on expression of junction-associated molecules and secretory function in rat Sertoli cells, Reprod. Toxicol. 30 (2010) 429-437.

[21] H. Fromme, A. Lainsch, A. Oddoy, et al., Elemental carbon and repairable particulate matter in the indoor air of apartments and nursery schools and ambient air in Berlin (Germany), Indoor Air 15 (2005) 335-341.

[22] G.W. Olsen, D.C. Mair, W.K. Reagen, et al., Preliminary evidence of a decline in perfluorooctanesulfonate (PFOS) and perfluorooctanoate (PFOA) concentrations in American Red Cross blood donors, Chemosphere 68 (2007) 105-111.

[23] L.W.Y. Yeung, M.K. So, G.B. Jiang, et al., Perfluorooctanesulfonate and related fluorochemicals in human blood samples from China, Environ. Sci. Technol. 40 (2006) 715-720. 